\title{
Evaluation of Accuracy of Drug Interaction Alerts triggered by Two Electronic Medical Record Systems in Primary Health Care
}

\author{
Rekha Gaikwad ${ }^{1}$, Ingrid Sketris², Michael Shepherd², Jack \\ Duffy ${ }^{3}$
}

\author{
${ }^{1}$ Dalhousie University, Halifax, Nova Scotia, gaikwad@cs.dal.ca \\ ${ }^{2}$ CHSRF/CIHR Chair in Health Services Research, Co-Sponsor: NSHRF, Professor, College \\ of Pharmacy, Dalhousie University, Halifax, Nova Scotia, Ingrid.sketris@dal.ca
}

${ }^{2}$ Professor, Faculty of Computer Science, Dalhousie University, Halifax, Nova Scotia, Shepherd@cs.dal,ca

${ }^{3}$ Professor, Management and Management and Information Sciences, Faculty of Management, Dalhousie University, Halifax, Nova Scotia.,j.duffy@ns.sympatico.ca

This paper presents the study done to evaluate the accuracy of drug interaction (DI) alerts triggered by two Electronic Medical Record (EMR) systems used in primary health care. Elderly patients commonly find it onerous to recollect their medications during medical interrogation. Physicians and pharmacists find it hard to keep abreast with the expanding medical and pharmacologic knowledge bases. Automated DI alerts are designed to make available evidence-based knowledge at the point of care. A Scenario-Based Software Architecture Analysis Methodology (SAAM) was used with interacting drug-drug pairs without patient medical histories, representing "hypothetical" patient scenarios to evaluate the drug-interacting quality attribute of two EMR systems. Three hundred and twenty-five drug-drug interaction (DDI) pairs were identified by a comprehensive literature search for common drugs used in the management of common conditions seen in the elderly population in primary health care. Three Reference programs were selected to determine the level of severity of drug interactions. A common severity rating scale was adapted due to variations in the DI severity scales used by the DI software programs and EMR systems in this study. Regression and correlation analyses were carried out on the DI alerts triggered by the two EMR systems and evaluated for their agreement with the Reference programs. True positive and true negative "severe" clinically significant DDI pairs were classified based on inclusion and exclusion criteria. Sensitivity, specificity and positive and negative predictive values were determined for the DI alerts. Low sensitivity $(26 \%, 33 \%)$, high specificity $(96 \%, 100 \%)$, positive $(0.286,1)$ and negative $(0.956,0.961)$ predictive values were obtained on evaluating the accuracy of DI alerts from two EMR systems. EMR systems evaluated in this research showed a limited potential to identify "severe" clinically significant DDls and considerable probability for triggering spurious alerts. This may be a reason for the overriding of DI alerts and interruption of the workflow of users of EMR systems. Reasons for the deficient performance of EMR systems can be due to unavailable updates or programming, database functioning discrepancies, and controversies in the clinical evidence or due to combination of these. 


\section{Keywords}

Clinical decision support, Drug-drug interaction, Electronic Medical Record system, Reference program

\section{Introduction}

The Canadian market has about 20,000 drug products and about 7000 drug interactions have been identified. Unrecognised drug interactions leading to adverse drug events occur due to inappropriate prescribing [1, 2, 3] which can lead to medication errors [4] and adversely affect patient safety and health outcomes.

Medication error reduction is an international issue [5] and is a growing health concern. The Institute of Medicine's report on medical errors indicated dangers inherent in the U.S. medical care system that might cause up to 98,000 deaths in hospitals and cost approximately $\$ 38$ billion per year [5]. The need to reduce inappropriateness and errors in the administration of prescribed medications has focused attention on the prevention of drugdrug interactions (DDIs). In recent years, the issue of adverse drug events resulting from DDIs has been highlighted by the national media, policy makers, and health care providers [6]. Patient wellbeing and better patient outcomes can be possible with high quality medical care that provides a patient-centered focus on medication safety.

\section{Methodology}

\subsection{Study Design}

During phase I, a narrative literature review was done using electronic journal resources (Journal of American Medical Informatics Association, International Journal of Medical Informatics, IEEE Journals, etc), PubMed's Medline and OVID to identify the uses, importance and implications of clinical decision support-based Electronic Medical Record (EMR) Systems in clinical practice and barriers to their implementation.

Phase II consisted of a comprehensive exploration of relevant resources to get an overview of the various types of EMRs available, methodologies used for their evaluation and quality attributes considered essential for implementation of EMR systems in primary health care. Phase III was comprised of another literature review done by reviewing various drug-related electronic and printed resources (Drug Information Resources) available on the Dalhousie University's College of Pharmacy's website <http://pharmacy.dal.ca/druginfo/index.html> in order to help create hypothetical patient scenarios in the form of drug-drug interaction pairs. This literature review was done with a view to identifying the potential errors in clinical practice (eg. medication, prescribing), the reasons for their occurrence and the areas where they can be prevented, common conditions and drugs prescribed in elderly patients in primary care, and common causes for the Adverse Drug Reactions (ADRs) and drug interactions seen in the elderly.

\section{2 “Object" Drug And "Precipitant" Drug Interaction Pairs}

An EMR system integrated with clinical decision support in the form of a knowledge base can be referred to as an information resource. The Scenario-Based Architecture Analysis Method (SAAM) [7] appeared in 1993, corresponding with the trend for a better understanding of general architectural concepts, as a foundation for proof that a software system meets more than just functional requirements. Scenario-based analysis is more concrete than the general definition of software quality [8] and is more context-sensitive. 
Scenarios can be used to represent software architecture evaluations qualitatively. In this study, we apply the questioning technique in the form of drug-drug interaction pairs analogous to "scenarios" $[9,10,11,12]$ without patient medical histories and employ them to evaluate the "alerting" quality attribute of electronic medical record system architecture to address the problem description of drug-drug interactions in elderly patients. The scenarios were created such that the EMR systems should trigger drug interaction alerts to signal against imminent drug-drug interactions possible with the combinations of medications that are commonly used in the elderly and aging patients in primary care.

The following resources were reviewed and searched for medications indicated in the primary care management of common conditions seen in elderly patients:

(a) Ontario Drug Therapy Guidelines [13] for:

- Chronic Heart Failure in Primary Care

- Stable Ischemic Heart Disease in Primary Care

- Osteoarthritis, Rheumatoid Arthritis, and Acute Musculoskeletal Injury

- The Management of Anxiety Disorders in Primary Care

- Peptic Ulcer Disease and Gastroesophageal Reflux

- The Pharmacotherapeutic Management of Diabetes Mellitus

- The Prevention and Treatment of Osteoporosis

(b) "Top Ten Dangerous Drug Interactions in Long-Term Care" available on Multidisciplinary Medication Management Project's website [14]

Drugs selected from the above resources to represent the "object drugs" [whose action is altered] by the "precipitant drugs" [which cause the altered action] were:

- Warfarin

- Acetylsalicylic acid (ASA)

- Atorvastatin

- Enalapril

- Naproxen

- Digoxin

- Clopidogrel

- Potassium

- Alendronate

- Hydrochlorothiazide

Precipitant drugs were adapted from the list of commonly prescribed medications from the "Top 50 Prescribed Medications, 2004" from the IMS Health Canada's Drug Monitor [15]. For the purpose of this study, if two or more brands of a chemical entity were in the top 50 medications, then they were noted by their generic names. The generic names of the drugs listed in "Top 50 Prescribed Medications, 2004" [15] were searched for their availability on the Drug Product Database (DPD) accessible from Health Canada's website [16]. Drugs indicated in the management of conditions identified in the literature review were selected for this study and drugs with hormonal combinations, inhalational and nasal use were excluded. A sample of 10 object drugs and 37 precipitant drugs was selected. The selected drugs were then arranged according to the World Health Organization's Anatomical Therapeutic Chemical (ATC) Index 2005 available on the World Health Organization Collaborating Centre for Drug Statistics Methodology resource [17].

\subsection{Determination Of Level Of Severity Of Drug Interaction}

Clinical decision support-based EMR systems function to support the clinical decisionmaking and dispensing process of physicians and pharmacists, respectively, by giving accurate automated drug interaction alerts. Any clinically significant drug-drug interaction is expected to be signalled by the EMR system at the point-of-prescription and point-ofdispensing, respectively. The rationale used during the selection of the drug-drug interactions

Proceedings of the $11^{\text {th }}$ International

Symposium on Health Information

Management Research - iSHIMR 2006 
pairs was that the clinical decision support tools integrated in the EMR systems are expected to give accurate and genuine drug interaction alerts for any drug-drug pair with a potential for interacting when combined. The level of severity of drug interaction for the combination of object drugs and precipitant drugs selected was determined individually using the following electronic drug information resources, referred to as "Reference" programs (RP1, RP2 and RP3) for this study:

- Micromedex ${ }^{\circledR}$ Healthcare Series. DRUG-REAX ${ }^{\circledR}$ Interactive Drug Interactions [18] (accessed July 2005 from Dalhousie University's College of Pharmacy) [clicked on drug interactions of all severities]

- Express Scripts DRUG DIGEST Check Interactions [19] (accessed July 2005 from Dalhousie University's College of Pharmacy's Drug Information Resources)

- MedScape for WebMD [20]: Multi-Drug Interaction Checker (accessed July 2005 from Dalhousie University's College of Pharmacy's Drug Information Resources)

The results determining the level of severity for each object drug-precipitant drug interaction pairs using each of the three Reference programs individually were tabulated according to the level of severity of drug-drug interaction. This gave a total of 334 drug-drug interaction pairs as indicated in Table 1. The absence of a drug interaction or, if present, the level of severity of interaction between the particular pair, issued for each drug-drug interaction pair on single input into the program was noted for the study.

Table 1: Number of object drug-precipitant drug interaction pairs selected for this study

\begin{tabular}{|l|l|}
\hline Object Drug & $\begin{array}{l}\text { Total object drug-precipitant } \\
\text { drug interaction pairs }\end{array}$ \\
\hline Warfarin & 36 \\
\hline Acetylsalicylic acid & 35 \\
\hline Atorvastatin & 34 \\
\hline Enalapril & 33 \\
\hline Naproxen & 37 \\
\hline Digoxin & 32 \\
\hline Clopidogrel & 31 \\
\hline Potassium & 37 \\
\hline Alendronate & 30 \\
\hline Hydrochlorothiazide & 29 \\
\hline Total & 334 \\
\hline
\end{tabular}

The 334 drug-drug interaction pairs were then used to obtain drug interaction alerts issued by a pharmacy EMR system and a physician's EMR system. The two EMR systems (EMR A and EMR B) are referred to as "user programs" in this study. The drug interaction alerts, triggered by both electronic medical record systems were identified and recorded using Microsoft Office Excel (2003). There were variations in the results obtained from the Reference programs determining the level of severity of drug-drug interactions and also in the drug interaction alerts triggered by EMR systems.

Variations were also noted in the drug interaction severity rating scales used by the Reference programs to determine the level of severity of DDI and by the two EMR systems to determine the level of severity of drug-drug interaction in the form of an "alert". Some use a severity rating scale ranging from "no" interaction, "mild" interaction, "moderate" interaction and "severe" interaction to describe the drug-drug interaction. Some use levels of severity to determine the clinical significance of the drug interaction in the form of rating from 1 to 5 where "1" denotes "severe drug-drug interaction" and " 5 " for "no" interaction. Some programs use a rating scale for measuring severity of the drug interaction denoted by " $A$ " for "No Known Interaction Data", "B" for "No Action Needed Data", "C" for "Monitor Therapy Data", "D" for "Consider Therapy Modification Data" and "X" to denote "Avoid Combination Data".

Proceedings of the $11^{\text {th }}$ International

Symposium on Health Information

Management Research - iSHIMR 2006 
A "common" severity rating scale for the level of severity of the drug-drug interactions was adapted for this study to translate results of the drug-drug interactions issued by programs in this study and to classify drug-drug interactions with respect to their level of severity. For this, rating scales used by different compendia were studied for their commonalities. Every compendium uses a slightly different rating system to classify drug-drug interactions [12, 21]. The interacting drugs in a drug-drug interaction are assigned to one of the following five classes (originally developed by the Drug Interaction Foundation) based on the need for an intervention to minimise the risk due to the interaction as mentioned in Hansten and Horn's Facts and Comparisons for Drug Interactions [22; pg. xiii]. These are shown in Table 2. Various studies $[6,9,12]$ have mentioned that different severity rating scales are used by various compendia to determine severity of drug-drug interactions as described in Table 3.

Table 2: Five classes of clinical significance of drug interaction as mentioned in Hansten and Horn's Facts and Comparisons for Drug interactions [22; pg. xiii]

\begin{tabular}{|l|l|}
\hline $\begin{array}{l}\text { Clinical } \\
\text { significance } \\
\text { numbers }\end{array}$ & DI and need for intervention to minimise the risk \\
\hline 1 & Avoid the combination $\rightarrow$ risk always outweighs the benefits \\
\hline 2 & $\begin{array}{l}\text { Usually avoid combination } \rightarrow \text { use the combination only under } \\
\text { special circumstance }\end{array}$ \\
\hline 3 & Minimise the risk $\rightarrow$ take action as necessary to reduce risk \\
\hline 4 & No action needed $\rightarrow$ risk of adverse outcomes appears small \\
\hline 5 & No interaction $\rightarrow$ evidence suggests no interaction \\
\hline
\end{tabular}

Table 3: Examples of severity rating scales for drug interactions used by different compendia [6, 9, 12]

\begin{tabular}{|l|}
\hline Description for severity rating scales for drug interactions used by some compendia \\
\hline Five-item rating scale (major, moderate, minor, none, not specified) \\
\hline Four-item summary measure (Code 1 : highly clinically significant; Code 2 : moderately clinically \\
significant; Code 3 : minimally clinically significant; Code 4 : not clinically significant) based on \\
three major factors: potential harm to the patient, frequency and predictability of occurrence, \\
and degree and quality of documentation \\
\hline Severity measure (major, moderate, minor) \\
\hline
\end{tabular}

For this study, "scale" has been defined as the set of response options ${ }^{12}$ from which one option is chosen to translate the result of the description of severity of the drug-drug interaction for a particular drug-drug combination. The "Common" severity rating scale for the drug-drug interaction pairs adapted for this study ranged from 1 to 4 according to their level of severity, their class for level of significance and the recommendations for prevention. The drug-drug interactions were rated from 1 to 4 with respect to the level of severity for the drugdrug interaction where "1" represented a "severe" drug interaction and "4" represented "no" drug interaction, as described in the Table 4 below.

The results obtained from the three Reference programs were translated using the "common" severity rating scale and compiled into a Microsoft Excel spreadsheet. In this study, one of the Reference programs (RP3) used two severity rating scales simultaneously to determine the level of severity of a particular drug-drug interaction. This program determined the level of severity in the form of description of the measure as "mild/ moderate/severe/no interaction" and in form of numbers (classes) ranging from " 1 to 5 " determining their level of severity per clinical significance. 
Table 4: "Common" severity rating scale adapted for this study to translate results of drug-drug interaction pairs determined by Reference programs and EMR systems

\begin{tabular}{|c|c|c|c|c|}
\hline $\begin{array}{l}\text { Rating } \\
\text { from } 1 \\
\text { to } 4\end{array}$ & $\begin{array}{l}\text { Severity } \\
\text { rating for DI }\end{array}$ & $\begin{array}{l}\text { Description for } \\
\text { severity rating }\end{array}$ & For EMR A & For EMR B \\
\hline 1 & $\begin{array}{l}\text { Severe } \\
\text { interaction } \\
\text { and classes } \\
\text { "1" and "2" }\end{array}$ & $\begin{array}{l}\text { - Avoid } \\
\text { administration of } \\
\text { combination }^{\ddagger}\end{array}$ & Severe & $\begin{array}{ll}\text { - } & X \rightarrow \text { Avoid } \\
\text { combination } \\
\text { - } & D \rightarrow \text { Consider } \\
& \text { therapy modification }\end{array}$ \\
\hline 2 & $\begin{array}{l}\text { Moderate } \\
\text { interaction } \\
\text { and class "3" }\end{array}$ & $\begin{array}{l}\text { Avoid } \\
\text { administration } \\
\text { unless it is } \\
\text { determined that the } \\
\text { benefit of } \\
\text { coadministration } \\
\text { outweighs the risk } \\
\text { to the patient }^{+}\end{array}$ & Moderate & $\begin{array}{l}\text { - } \rightarrow \text { Monitor therapy. } \\
\text { Data demonstrate } \\
\text { that the specified } \\
\text { agents may interact } \\
\text { with each other in a } \\
\text { clinically significant } \\
\text { manner. The } \\
\text { benefits of } \\
\text { concomitant use of } \\
\text { these two } \\
\text { medications usually } \\
\text { outweigh the risks. }\end{array}$ \\
\hline 3 & $\begin{array}{l}\text { Mild } \\
\text { interaction } \\
\text { and class "4" }\end{array}$ & $\begin{array}{l}\text { - Minimize risk by } \\
\text { considering } \\
\text { alternative agents } \\
\text { or change dosage } \\
\text { or route of } \\
\text { administration* } \\
\text { No action } \\
\text { needed } \rightarrow \text { risk of } \\
\text { adverse outcomes } \\
\text { appears small }\end{array}$ & $\begin{array}{l}\text { Not issued for } \\
\text { any of drug- } \\
\text { drug interaction } \\
\text { pair in this } \\
\text { study }\end{array}$ & $\begin{array}{l}\mathrm{B} \rightarrow \text { No action } \\
\text { needed }\end{array}$ \\
\hline 4 & $\begin{array}{l}\text { No } \\
\text { interaction } \\
\text { and class "5" }\end{array}$ & $\begin{array}{ll} & \text { No interaction } \rightarrow \\
\text { evidence suggests } \\
\text { no interaction }\end{array}$ & $\begin{array}{ll}- & \text { No drug } \\
\text { interaction } \\
\text { alert } \\
\text { - } \quad \text { Duplicate } \\
\text { therapy } \\
\text { alert }\end{array}$ & $\begin{array}{ll} & \mathrm{A} \rightarrow \text { No known } \\
\text { interaction } \\
\text { - } & \text { No interaction alert }\end{array}$ \\
\hline
\end{tabular}

The "common" severity rating scale was created by considering the commonality of rating scales used by different compendia as mentioned in Table 2 and Table 3. DI - Drug interaction; ${ }^{\dagger}$ - The risk of adverse patient outcome precludes concomitant administration as risk always outweighs the benefits, usually avoid combination $\rightarrow$ use combination only under special circumstance; ${ }^{+}$- Use the combination only under special circumstance, needs close patient monitoring, minimise risk and take action as necessary to reduce risk; * - Risk of adverse outcomes appears small and patient monitoring is suggested

The "common" severity rating scale used for translating the results of the severity rating in this study was used to rate the "description" of severity as well as the "level" of severity, determined for individual drug-drug interaction pairs by RP3. Translation of the results of severity measures determined by RP3 into respective rating using the "common" severity rating scale for this study did not alter the mean and the variance of results obtained from RP3. Correlation analysis was done on the "translated" results (using "common" severity rating scale) of determination of levels of severity of drug interactions for the three Reference programs. This analysis $(0.48,0.56$ and 0.54$)$ showed that the three Reference programs reasonably agreed with each other. This analysis gave concurrent validity and acceptability

Proceedings of the $11^{\text {th }}$ International

Symposium on Health Information

Management Research - iSHIMR 2006 
for usage of "common" severity rating scale for this study. Out of the 334 drug-drug interaction pairs, 9 pairs, amitriptyline with acetylsalicylic acid, atorvastatin, naproxen, digoxin, clopidogrel, potassium, alendronate, hydrochlorothiazide and enalapril, respectively, were deleted because the EMR B showed a message "the drug is unavailable for interaction referencing" for these pairs. This resulted in a sample of a total of 325 object drug-precipitant drug interaction pairs for this study.

\section{Statistical Analysis}

In this study, reliability which is the property of consistency of a measurement that gives the same result on different occasions [23] is an estimate of agreement between the three Reference programs. This was determined using Cronbach's $\alpha$. Cronbach's $\alpha$ is the most widely used index of the reliability of a scale [24] and it provides an estimate of how well the Reference programs measure the severity of drug-drug interaction pairs. It is also the most common form of inter-rater reliability coefficient. The Statistical Package for the Social Sciences (SPSS) 11.5.1 version for Windows was used to determine the Cronbach's $\alpha$. A Cronbach's Alpha of 0.7592 was obtained which was an acceptable estimate of reliability of the measure of level of severity of drug interaction for the three Reference programs. A reliability coefficient of 0.80 or higher is considered as "acceptable" in most Social Science applications [25]. By convention, alpha should be 0.70 or higher to retain an item in a scale [26]. Therefore, we are justified in combining the three Reference programs into one summary Reference program by adding each drug-drug interaction rating together and dividing by 3 .

The drug interaction alerts triggered by the two EMR systems when tested with drug-drug interaction pairs to signal the severity of drug-drug interaction were translated into representative severity levels using the "common" severity rating scale adapted for this study as shown in Table 4 above. The results obtained by testing for the level of severity of drugdrug interaction pairs issued by the three Reference programs and their collective average were considered as the "dependent or response" variables and those issued for the same drug-drug interaction pairs by the user programs (EMR A and EMR B) as "independent or predictor" variables. Simple and multiple regression analyses were carried out on the resulting data compiled using SPSS. Simple regression was done using each of the Reference programs and their average as the response variable and each of the user programs (EMR A and EMR B) as the predictor variable. Multiple regression was done using each Reference program and its average as response and both the user programs as predictor variables.

The objective of performing regression analysis on the set of data was to ascertain the probable form of relationship between the variables [27; chapter 9] in this study. Regression analysis was done which gave $R$-squared $\left(R^{2}\right)$, an estimate of the variation in the Reference program measure explained by the regression. R-squared is also called the "Coefficient of determination" and can assume values from 0 (none of the variation in the dependent variable is explained by the regression) to 1 (all the variation in the dependent variable is explained by the regression) [27; chapter 9]. The regression analysis also gave an intercept which gave the adjustment for scale differences and a slope which gave the weight of the user program in predicting the measure given by the Reference program. It also gave the Analysis of Variance (ANOVA) which interpreted the significance of the regression equation in explaining the variability in the measure of the Reference program. A significant value of the $\mathrm{F}$ statistic means that the $\mathrm{R}^{2}$ is significantly different from zero.

The value of $R^{2}$ obtained from simple regression ranged from .227 to .847 with maximum for Reference program 3 versus EMR A system and minimum for Reference program 3 versus EMR B system. This showed that the agreement between the EMR systems and the Reference programs varied between $22.7 \%$ and $84.7 \%$. The $\mathrm{R}^{2}$ obtained from multiple

Proceedings of the $11^{\text {th }}$ International

Symposium on Health Information

Management Research - iSHIMR 2006 
regression analysis ranged from .412 to .847 with maximum for the Reference program 3 and both EMR systems and minimum for the Reference program 1 and both EMR systems. A stepwise regression with $F$ value set as "F-to-enter $>=3.840$, F-to-remove $<=2.710$ " was then carried to find out which of the two EMR systems gets entered first. This estimated which one of the two EMR systems issued results (alerts) which had the most alignment and agreement with which one of the Reference programs. The results obtained by stepwise regression showed that EMR A was always entered first and EMR $B$ was excluded at all steps. The results concluded that the EMR system $A$ was a better predictor than the EMR system B for all the Reference programs as well as their average. Also, EMR A had more alignment with Reference program 3 as it was the only predictor variable "entered" during stepwise regression with Reference program 3.

\section{Evaluation Of Accuracy Of EMR Systems}

Accurate drug interaction alerts referred to the "genuine" drug interaction alerts issued by the EMR systems to identify clinically significant drug-drug interactions (DDI) pairs for this study. The combination of drugs which when tested with the Reference programs gave a "major", "severe" and/ or severity levels "1" or "2" with any of the Reference programs represented the clinically significant drug interaction pair. The inclusion and exclusion criteria for clinically significant and not clinically significant drug-drug interaction pairs are listed in Table 5. A true positive (TP) drug-drug interaction (DDI) pair has been defined for this study as a "clinically significant DDI pair" and a true negative (TN) DDI pair as a "not clinically significant DDI pair". The EMR systems in this study were expected to trigger genuine drug-drug interaction alerts for the clinically significant (true positive) DDI pairs selected for the study and ignore the not clinically significant (true negative) DDI pairs.

Table 5: Inclusion and exclusion criteria used for selection of true and false positive and true and false negative drug-drug interaction pairs

\begin{tabular}{|l|l|l|}
\hline Criteria & $\begin{array}{l}\text { Clinically significant Or } \\
\text { True Positive DDI pairs }\end{array}$ & $\begin{array}{l}\text { Not Clinically significant Or } \\
\text { True Negative DDI pairs }\end{array}$ \\
\hline Inclusion & $\begin{array}{l}\text { Any DDI pair determined to be } \\
\text { "Severe", "Major" and/ or belong } \\
\text { to severity level "1" or "2" by any } \\
\text { one of the Reference programs }\end{array}$ & $\begin{array}{l}\text { Any DDI pair determined to have } \\
\text { "No" drug interaction by all three } \\
\text { Reference programs }\end{array}$ \\
\hline Exclusion & $\begin{array}{l}\text { Any DDI pair determined to have } \\
\text { "No" drug interaction by all three } \\
\text { Reference programs }\end{array}$ & $\begin{array}{l}\text { Any DDI pair determined to be } \\
\text { "Severe", "Major" and/ or belong to } \\
\text { severity level "1" or "2" by any one } \\
\text { of the Reference programs }\end{array}$ \\
\hline
\end{tabular}

Note: $\mathrm{DDI}=$ Drug-Drug Interaction

Following the inclusion and exclusion criteria, a sample consisting of 15 true positives ("clinically significant") and 247 true negatives ("non-clinically significant") was selected. Drug-drug interaction alerts were classified for this study as illustrated in Table 6 . They were classified into true positive, false positive, true negative and false negative drug-drug interaction alerts.

\subsection{Sensitivity And Specificity Of Drug Interaction Alerts}

Sensitivity: For this study, sensitivity for an EMR system was defined as the number of true positive drug-drug interaction alerts given by the system for all the true positive DDI pairs selected for this study.

Proceedings of the $11^{\text {th }}$ International

Symposium on Health Information

Management Research - iSHIMR 2006 
Sensitivity $=$ Number of true positive DI alerts

Number of true positive DI alerts + false negative DI alerts

Specificity = For this study, specificity for EMR system was defined as the number of true negative DDI pairs identified by the system among all the true negative DDI pairs selected for this study.

Specificity $=$ Number of true negative DI alerts

Number of true negative DI alerts + false positive DI alerts

Table 6: Classification of drug interaction alerts into true and false positive and into true and false negative, respectively

\begin{tabular}{|l|l|}
\hline Classification of DDI alerts & Definition for this study \\
\hline True positive (TP) DDI alerts & $\begin{array}{l}\text { Genuine DDI alert for a clinically } \\
\text { significant DDI pair }\end{array}$ \\
\hline False positive (FP) DDI alerts & $\begin{array}{l}\text { Spurious DDI alert for a not clinically } \\
\text { significant DDI pair }\end{array}$ \\
\hline True negative (TN) DDI alerts & $\begin{array}{l}\text { No DDI alert for a not clinically significant } \\
\text { DDI pair }\end{array}$ \\
\hline False negative (FN) DDI alerts & $\begin{array}{l}\text { No or spurious DDI alert for a clinically } \\
\text { significant DDI pair }\end{array}$ \\
\hline
\end{tabular}

Note: DDI = Drug-Drug Interaction

Positive predictive value (PPV): For this study, positive predictive value is the probability that an alert is triggered by the EMR system for a true positive (clinically significant DDI pair). PPV $=\underline{\text { Number of true positive DI alerts }}$

Number of true positive DI alerts + false positive DI alerts

Negative predictive value (NPV): For this study, negative predictive value is the probability that a drug-drug interaction alert is not triggered by the EMR system for a true negative (not clinically significant DDI pair).

$\mathrm{NPV}=$ Number of true negative DI alerts

Number of true negative DI alerts + false negative DI alerts

Table 7 illustrates the results of calculation of sensitivity, specificity, PPV and NPV for EMR A and EMR B.

Table 7: Estimation of accuracy of EMR systems

\begin{tabular}{|l|l|l|}
\hline Evaluation & EMR A & EMR B \\
\hline TP DDI alerts & 5 & 4 \\
\hline TN DDI alerts & 247 & 237 \\
\hline FP DDI alerts & 0 & 10 \\
\hline FN DDI alerts & 10 & 11 \\
\hline Sensitivity & $5 / 15=0.333$ & $4 / 15=0.267$ \\
\hline Specificity & $247 / 247=1$ & $237 / 247=0.959$ \\
\hline PPV & $5 / 5=1$ & $4 / 14=0.286$ \\
\hline NPV & $247 / 257=0.961$ & $237 / 248=0.956$ \\
\hline
\end{tabular}

Note: EMR - Electronic Medical Record system, TP - True Positive, TN - True Negative, FP - False Positive, FN - False Negative

\section{Results}

The regression analyses showed that drug-drug interaction alerts issued by EMR A better agreed with drug-drug interaction severity results determined by Reference program 3 than

Proceedings of the $11^{\text {th }}$ International

Symposium on Health Information

Management Research - iSHIMR 2006 
those issued by EMR B. The Crohnbach's alpha of 0,7592 for the three Reference programs used for this study demonstrated a fairly reasonable agreement with respect to their determination of level or description of rating of severity of drug-drug interaction, though there can be uncertainty of evidence related to drug-drug interactions in some cases.

Out of the 15 clinically significant drug-drug interaction combinations selected for this study, the EMR A accurately identified 5 drug-drug interaction combinations (approximately 33\%) as "severe" and did not accurately identify 10 combinations (approximately 67\%) as "severe". Similarly, the EMR B accurately identified 4 out of 15 (approximately 27\%) clinically significant drug-drug interaction combinations selected for this study as "severe" and did not accurately identify 11 combinations (approximately 73\%) as "severe". The evaluation of accuracy for two EMR systems by estimating their sensitivity and specificity resulted in low sensitivity (0.27 and 0.33 ) and excellent specificity (1.0 and 0.96$)$ estimates to identify drugdrug interactions. The low sensitivity for both EMR systems suggested that they did not issue accurate drug-drug interaction alerts to identify clinically significant drug-drug interactions as "severe" for more than $67 \%$ of the combinations. Conversely, higher estimates for specificity demonstrated that the two EMR systems ignore the "not clinically significant" drug-drug interaction pairs well.

The estimation of positive and negative predictive values for EMR A, 1.0 and 0.96, respectively, showed that when they issued a drug-drug interaction alert, it was accurate.

\section{Discussion}

Due to the complex workflow of physicians and pharmacists and the constantly expanding medical and pharmacologic knowledge bases, there is the potential of drug interactions being unrecognized. The large number of drugs available in the Canadian market and the drug interactions among them add to this risk for patient safety at the point of prescribing. Physicians and pharmacists are expected to prescribe and dispense, and counsel on, respectively, the most appropriate medications which can produce the most optimal benefits to the patients based on their judgments, knowledge and experience. Drug information resources available at the point of care play a crucial role in the identification of imminent drug interactions, and can safeguard against medication errors due to inappropriate prescribing and adverse drug reactions. This study evaluated the variability in the severity rating scales used by various drug information resources to determine levels of severity of drug-drug interaction pairs used in this research. Over-alerting by the EMR systems against drug-drug interaction alerts was not significantly revealed from this study, though inaccurately alerting against "severe" drug-drug interactions was evident from the sensitivity (0.27 to 0.33 ), specificity (1.00 to 0.96$)$, positive predictive (1.00 and 0.29$)$ and negative predictive $(0.96$ and 0.956$)$ values for the two EMR systems, respectively.

Various drug interaction software evaluation studies [9, 28, 29] used drug-drug interaction pairs and different methodologies to analyze performance of drug information resources. This study evaluated the accuracy of the drug interaction alerts and performance of EMR systems and also assessed the variations in the severity rating scales used by electronic drug information resources.

Hazlet et al. [9] performed a one-time performance test of the pharmacy computer systems in Washington State to accurately identify clinically significant drug interactions. In their study, 6 patient profiles with 37 drug-drug interaction pairs were used to evaluate the performance of drug interaction software installed in 516 pharmacy chains and health maintenance organization pharmacies. They identified in their study the complexity of pharmacy software systems, their inability to detect one-third of clinically important drug interactions and the variability in their results. They found the sensitivity of the software programs to range from 0.44 to 0.88 , with 1.00 being perfect and specificity to range from 0.71 to 1.00 . The positive 
predictive values ranged from 0.67 and 1.00 and negative predictive value ranged from 0.69 to 0.90 .

In 2001, Abarca et al. [6] conducted a cross-sectional one-time evaluation study in the United States of the agreement among drug-drug interaction compendia to designate interactions as having greatest clinical importance "major" drug-drug interactions. Some of the drug interaction compendia evaluated in their study were used as Reference programs for this research. They identified that of the 406 major drug-drug interactions listed in one or more of the four compendia in their study, only $9(2.2 \%)$ of these major drug-drug interactions were listed in all four compendia. They found an intra-class correlation coefficient of -0.092 (1.0 indicating perfect agreement among the compendia and 0 indicating no agreement) which indicated a low agreement between the four compendia on the classification of major drug interactions and more variation within the compendia than that among the compendia. Their study also mentioned that computer software vendors should focus on drug interaction alerts to signal against clinical significant drug interactions to enhance the effectiveness of drug information resources in physicians' and pharmacists' decision making.

Though the drug-drug interaction alerts can be customized to identify only "clinically important" drug interactions in software systems, this raises a possibility of missing drug interactions causing adverse drug reactions if the drug interaction databases differ in their severity rating scales. The sensitivity, specificity, positive predictive and negative predictive values obtained in this research, imply that if the end user of the EMR system decided to suppress the spurious drug interaction alerts, allowing the systems to signal only against the "severe" drug-drug interactions, then the possibility of missing clinically important or "severe" drug-drug interactions would increase.

Important drug information with regard to drug interactions may be missing from drug interaction databases, as concluded by Smith et al. [29] in their study to evaluate the ability of a common drug interaction database to identify clinically important drug interactions involving drugs used in transplant medications from the prescriber's perspective. The drug interactions used in their analysis were generated from an expert panel, a common drug interaction database and two standard drug interaction references. Their study concluded that the drug interaction failed to identify approximately $70 \%$ of drug interactions considered significant by the expert panel and more than $85 \%$ of alerts generated were considered clinically significant. In their study, "the expert panel agreed on the clinical significance of the 272 interactions when the interactions were evaluated independently" [29] and there was a marginal first-pass agreement among the 3 experts indicated by kappa of 0.3526 . They also found that the drug interaction database was most deficient in identifying interactions resulting from additive toxicity.

If the integrity of the information populating the drug interaction databases is improved, the discrepancies in the programming and assumptions made at the core development of the databases and software systems are dealt with effectively, the drug interaction alerts can play a vital role in signalling potential drug interactions.

\section{Limitations}

This study was conducted as a single-input, single-performance evaluation process and the data were collected and coded by the same single researcher. The drugs identified for this study to represent the "object" and "precipitant" drugs were selected on the basis of statistical information and literature available with relation to elderly patients seen in primary health care. We did not examine the primary literature related to drug-drug interactions or conduct a focus group of experts to determine the clinical relevance of the drug interactions. This study involves only a subset of possible drug interactions with the large number of prescription drugs available in Canada and their drug-drug interactions. There may have been 
unidentified clinically significant drug-drug interaction combinations which could have substantially changed the results.

The study involved determination of level of severity of drug-drug interactions using only three drug information resources. Inclusion of additional drug information resources for the determination of severity levels could have altered the results. The cut-off point for drug-drug interactions to be rated as "severe" and not "moderate" or "minor" was with the perspective that the drug-drug combination, if prescribed, results in risks outweighing the benefits to the patients. Expansion of this cut-off point to include "moderately" severe drug-drug interactions could have substantially changed the results.

\section{Conclusions}

The results obtained in this study showed that EMR systems vary in their identification of clinically significant drug-drug interactions for elderly patients in primary health care and cannot be used as the sole source of information. Pharmacists and physicians should not completely rely on the EMR systems to trigger clinically significant drug-drug interaction alerts and signal against adverse drug events. The study also suggested that EMR systems give a large number of spurious automated drug-drug interaction alerts which can be annoying to busy physicians and pharmacists because they interrupt their workflow. The performance of the software is influenced by the unavailability of updates or relevant drug interaction information (data) in drug interaction databases; discrepancies in the software itself and/or in the drug interaction databases; programming incongruity; or a combination of these reasons.

The education of physicians and pharmacists about the mechanisms and significance of drug interactions is critical. This study also illustrated that there was no universality in the severity rating scales for drug interactions used by the drug information software programs. The difference in severity rating scales for drug interactions used by different drug information databases can make it difficult for EMR systems to reliably support clinical decision-making and the dispensing process at the point of care for physicians and pharmacists. This study suggests that EMR systems should be updated, evaluated and studied on a regular basis for their accuracy and performance to identify potential drug interactions and realize the promise of improving patient outcomes. The expectations for drug interaction alerts and their desirable levels vary between the end-users of the EMR systems and this deserves further research into the user-specific needs regarding the alerting.

\section{Acknowledgements}

Rekha Gaikwad acknowledges the salary support received from the Canadian Health Services Research Foundation (CHSRF), the Canadian Institutes of Health Research (CIHR) and the Nova Scotia Research Foundation (NHSRF) and the CIHR PhD/PostDoc Strategic Training Program (CHPSTP). She acknowledges Lisa Napier from the Nova Scotia Department of Health and Jane Gillis and Kim Sponagle from the College of Pharmacy for facilitating access to the software programs.

\section{References}

[1] Tamblyn R, Huang A, Perreault R, et al. The Medical Office of the 21st Century (MOXXI): Effectiveness of Computerized Decision-Making Support in Reducing Inappropriate Prescribing in Primary Care. CMAJ. 2003; 169(6):549-556.

[2] Hannan TJ. Detecting Adverse Drug Reactions to Improve Patient Outcomes. Int J Med Inform. 1999; 55(1):61-64.

Proceedings of the $11^{\text {th }}$ International 
[3] MacKinnon NJ. Improving the Management of Medication Use in Older Adults. (editorial) Geriatrics Today: Journal of the Canadian Geriatrics Society. 2002; 5: 63-5.

[4] National Coordinating Council for Medication Error Reporting and Prevention. Availaible on <http://www.nccmerp.org/aboutMedErrors.html>. Accessed June 2005

[5] Ash JS, Berg M, Coiera E. Some Unintended Consequences of Information Technology in Health Care: The Nature of Patient Care Information System-Related Errors. J Am Med Inform Assoc. 2004; 11(2):104-112.

[6] Abarca J, Malone DC, Armstrong EP, et al. Concordance of Severity Ratings Provided in Four Drug Interaction Compendia. J Am Pharm Assoc (Wash DC). 2004; 44(2):136-141.

[7] Dobrica L. and NiemelaĖ E., Member, IEEE Computer Society: A Survey on Software Architecture Analysis Methods. IEEE TRANSACTIONS ON SOFTWARE ENGINEERING. July 2002; 28 (7).

[8] Morgan S, Bassett K, Mintzes B. Outcomes-Based Drug Coverage in British Columbia. Health Aff (Millwood). 2004; 23(3):269-276.

[9] Hazlet TK, Lee TA, Hansten PD, Horn JR. Performance of Community Pharmacy Drug Interaction Software. J Am Pharm Assoc (Wash). 2001; 41(2):200-204.

[10] Fernando B, Savelyich BS, Avery AJ, et al. Prescribing Safety Features of General Practice Computer Systems: Evaluation using Simulated Test Cases. BMJ. 2004; 328(7449):1171-1172.

[11] Sintchenko V, Coiera E, Iredell JR, Gilbert GL. Comparative Impact of Guidelines, Clinical Data, and Decision Support on Prescribing Decisions: An Interactive Web Experiment with Simulated Cases. J Am Med Inform Assoc. 2004; 11(1):71-77.

[12] Malone DC, Abarca J, Hansten PD, et al. Identification of Serious Drug-Drug Interactions: Results of the Partnership to Prevent Drug-Drug Interactins. J Am Pharm Assoc (Wash DC). 2004; 44(2):142151.

[13] The Center for Evaluation of Medicines. Resources. Ontario Program for Optimal Therapeutics. 2000. Availaible on <http://www.thecem.net/resourcesframe.html>. Accessed July 2005.

[14] Multidisciplinary Medication Management Project. Ten Dangerous Drug Interactions in Long-Term Care. Available on <http://www.scoup.net/M3Project/topten/index.htm>. Accessed July 2005.

[15] IMS Health Canada. IMS DRUG MONITOR: CANADA. Top 50 Prescribed Medications*, 2004. Available on <http://www.imshealthcanada.com/htmen/3_2_14.htm>. Accessed June-July 2005.

[16] Health Canada. Drug Product Database (DPD). Active Products Search. Available on <http://search.hc-sc.gc.ca/cgi-bin/query?mss=dpd/english/active/simple>. Accessed July 2005.

[17] World Health Organization Collaborating Centre for Drug Statistics Methodology. ATC/DDD Index 2005. Available on <http://www.whocc.no/atcddd/>. Accessed in August 2005.

[18] Micromedex (R) Healthcare Series. DRUG-REAX $®$ Interactive Drug Interactions. Accessed July 2005 from Dalhousie University's College of Pharmacy

[19] EXPRESS SCRIPTS DRUG DIGEST Check Interactions. Available on <http://www.drugdigest.org/DD/Interaction/ChooseDrugs/1,4109,,00.html>. Accessed in July 2005 from Dalhousie University's College of Pharmacy's Drug Information Resources

[20] MedScape for WebMD. Multi-Drug Interaction Checker. Available on

$<$ http://www.medscape.com/druginfo/druginterchecker?cid=med\&monotype=druginteractions\&drugid= \&drugname>. Accessed in July 2005 from Dalhousie University's College of Pharmacy's Drug Information Resources.

[21] Juurlink DN, Mamdani M, Kopp A, Laupacis A, Redelmeier DA. Drug-Drug Interactions among Elderly Patients Hospitalized for Drug Toxicity. JAMA. 2003; 289(13):1652-1658.

[22] Hansten PD, Horn JR. Hansten and horn's drug interactions, analysis and management. 1997; updated January 2005

[23] McBurney DH, White TL. Research Methods. Sixth Edition ed. 2004.

[24] Streiner DL. Starting at the Beginning: An Introduction to Coefficient Alpha and Internal Consistency. J Pers Assess. 2003; 80(1):99-103.

[25] UCLA Academic Technology Services. Stat Computing. SPSS.FAQ. Available at <http://www.ats.ucla.edu/stat/spss/faq/alpha.html>. Accessed July-August 2005.

[26] Reliability. Available at <http://www2.chass.ncsu.edu/garson/pa765/reliab.htm>. Accessed July 2005.

[27] Daniel WW. Biostatistics: A Foundation for Analysis in the Health Sciences. 7th ed. New York: Wiley, 1999.

[28] Kimberly A. Galt e. al.: Personal digital assistant-based drug information sources: potential to improve medication safety: J Med Libr Assoc 93(2) April 2005 
[29] Wendy D Smith, Randy C Hatton, Amy L Fann, Maher A Baz, and Bruce Kaplan: Evaluation of Drug Interaction Software to Identify Alerts for Transplant Medications: Ann Pharmacother 2005; 39:45-50; Published Online, 14 Dec 2004, DOI 10.1345/aph.1E331

Proceedings of the $11^{\text {th }}$ International 